Send your letters to the editor, British Dental Journal, 64 Wimpole Street, London W1G 8YS E-mail bdj@bda dentistry.org.uk

Priority will be given to letters less than 500 words long. Authors must sign the letter, which may be edited for reasons of space

\section{Dentistry- a medical specialisation?}

Sir,- Writing from distant Nepal, Dr M. D. Bhatterai (BDJ 2003; 194: 466) raises some pertinent points and an insight into the philosophy of the training pathway for dentists in the UK which has also long been a personal issue for me. Dentists as a separate profession are really an anachronism, and we stand where we are today vis a vis the wider medical profession entirely due to a decision made around two centuries ago by the luminaries of the time to split away from mainstream medical training.

Were we to apply the organisational skills, planning ability and educational requirements of today in setting up training programmes from scratch for people such as obstetricians, proctologists ophthalmologists and dennatologists (to name a selected few) would we train each as a separate profession? Highly unlikelywhy should the management of disorders of one particular part of the body necessitate a separate and discreet profession trained in parallel with those that look after other parts of the body, and sometimes its entirety? The people skills and diagnostic whole person information needed varies not one bit. We use similar adjunctive aids to this diagnosis and management - radiography, CT scans, histology and many others. That dentists enjoy parity in career grades and salary in the hospital service, the armed forces and academia (but for some reason not the GDS!) reflects this.

Yet what we see today with regard to dentists flies in the face of all this. The overlap with the medical course is huge, with dental and medical students sharing the same lecture theatres and seminar rooms in subjects from anatomy and neurology through many aspects of human disease. Cost effective it cannot be.

The results are dentists who are over trained for treating the basic oral diseases (caries and periodontal) that are still widely evident in the British public. Disease control that could often be effectively delivered by therapists is subjugated beneath the pressure to deliver in the NHS - in high volume in order to survive - low cost, low quality reparative technical exercises often involving crown and bridgework that often compound an already poor situation for appropriate diagnosis and management in a time starved environment. Similarly, the current complex and long pathway to seniority in oral and maxillofacial surgery for instance, is a product at the other end of the spectrum of replication, and perhaps could then be the subject of some moves towards rationalisation.

We have surely moved on in our mindset and can be proud of the ongoing research and the growing understanding we have of the oral environment. The immense diagnostic, therapeutic reconstructive and rehabilitative science that drives our professional care in helping our patients is aligned to this.

The task of realigning comprehensive oral healthcare with the rest of medicine as a speciality that could be followed after completing a basic medical degree for all 'doctors' would be huge. It would of course have a tremendous knock on effect for institutions, jobs and established empires. I sincerely hope nevertheless it is an evolution that will follow in the not to distant future, and that we finally get to leave behind the legacy of such as John Tomes and other giants of our early development who arguably with many at that time were no doubt responding to the perceived technical needs of the day.

\section{K. Marshall}

\section{Surrey}

Sir,- I read M.D. Bhattarai's letter with great interest. As a dental graduate currently in my third year at medical school, I felt the need to comment.

I agree that it is important for dental students to have a broad and sound knowledge of basic medicine and surgery. However, I disagree with M. D. Bhattarai's assumption that undergoing basic medical training will help dental students to 'consider the whole person'. Having a broader knowledge of medicine will not necessarily equip dental students better.
However, a sound understanding of biomedical sciences and human disease (medicine and surgery) appropriate for dentistry, good communication skills, development of attitudes and behaviours befitting the profession, appreciation of the diagnosis, management, treatment and prevention of dental disease and the development of adequate technical skills will. I fear that a basic medical course leading into dentistry would do dental education as it stands, and the practice of dentistry, a great disservice.

I thoroughly enjoyed my five years at dental school and deeply appreciated the early patient contact and treatment of cases, the continuity of patient care and the personal feedback, support and protection the smaller dental faculty provided. I feel these are important and unique aspects of undergraduate dental education and an 'integrated' medically based course would dilute these essential features.

In the current climate of mounting student debt and planned increases in tuition fees, an extended course would discourage many suitably qualified and motivated students from applying for a medically based dental course. I believe that most UK dental schools adequately meet the standards set out by the General Dental Council in terms of taught courses in biomedical sciences and human disease.

Under the current educational system, most dental graduates will have acquired adequate diagnostic skills, are able to recognise signs and symptoms of common systemic disease, and can manage medical emergencies that may present in a dental surgery. They are aware of and able to forward referrals to specialists with dual qualifications based in oral medicine or maxillofacial surgery for the management of patients with complex medical conditions and problems.

Perhaps, more importantly, we should be addressing the issue of undergraduate medical courses failing to highlight the importance of orofacial manifestations of systemic disease, allocating little or no time in their curricula to rectify this oversight. This does not necessarily mean 
that medical students have to undergo a

five year dental course!

Rather than completely overhauling the current system of undergraduate dental education, more training and consultant posts ought to be created in dually based specialities such as oral medicine and maxillofacial surgery to acknowledge the interface between medicine and dentistry. These departments are usually involved in the teaching of human disease (medicine and surgery) to dental students and it therefore becomes important that these specialities be supported and expanded. B. P. Rajlawat Glasgow

\section{Tooth notation confusion}

Sir,- I read with disbelief the notice 'A change in recording tooth notation' that was printed in BDJ 2003; 194: 387. Why all the mental gymnastics with the Palmer tooth notation? This method was thrown out about two decades ago in the US when computers came of age. We now use the designation \# 1-32 for permanent teeth and A-T for deciduous teeth in all communications among dentists, insurance companies, government agencies, etc. It eliminates any possibility of confusion when referring to a particular tooth and is so much simpler with less risk of mistakes. For the examples given in the Journal notice, UR7(17) becomes \#2 and LL5(35) becomes \#20. This 'tradition' is not an asset. Come join us in the 21st Century.

\section{G. Belok}

\section{New Jersey}

\section{Older patients}

Sir,- We read with interest the paper by Professor Tinker (BDJ 2003; 194: 369-372) concerning the increases in numbers and percentages of older people in the UK population. In this respect we feel that some work recently carried out by the Dental Practice Board and the University of Birmingham can add to the discussion of what this means for dentistry. The Dental Practice Board administers a database for the payment of all GDS dentists in England and Wales from which detailed treatment data have been retained, extending back more than eleven years. This archive of data has facilitated detailed analysis of treatment trends and rates of intervention and reintervention. In particular we have analysed the association between patient age and re-intervention within one year of placement of direct restorations, where re- intervention may be by replacement of the restoration by one of the same type or different type of restoration, by crowning, by extraction or by other treatments. The figure, based on over 100,000 restorations placed in the year 2000, illustrates that restorations placed in older patients are more likely to receive re-intervention within one year than restorations placed in younger patients. Indeed, the one-year re-intervention rate increases with patient age, from about $6 \%$ at age 25 years to $16 \%$ at age 65 years.

Professor Tinker's paper has informed us that there will be an increase in overall numbers of older people in future and that they retain their teeth longer. If that is the case, then our findings indicate that the percentage and number of early reinterventions will rise, with consequential increase in the number of unplanned attendances. This may have implications for practising dentists in the future, especially if they are treating large numbers of older patients.

\section{P. S. K. Lucarotti \\ R. L. Holder}

F. J. T. Burke

\section{Contaminated gloves}

Sir,- I have been reminded of some of routine procedures which we unquestioningly adopt in the name of cross-infection control and health and safety precautions. I refer to the use of heavy-duty rubber gloves to protect our staff from the hazards associated with cleaning dirty instruments before sterilization. The hazards relate to puncture wounds of the skin in the presence of the contaminants: potentially infective blood, saliva and tissue fragments; irritant or toxic materials like etch gel, uncured resin and amalgam.

The problem which arises in practice is that the heavy duty gloves become contaminated in use. The question then is how is it possible for my nurse to safely store, use and re-use such heavily contaminated gloves? There is no published description of the safe technique for donning a contaminated glove. These heavy duty gloves do not provide effective protection from punctures by sharps - even armoured brick-layers gloves cannot do that - and if they are only of use as a barrier to the usual surface contaminants why not continue to use the procedure gloves which apparently provided adequate protection for my nurse until the patient got out of the chair?

Could we not re-think the statement 'Heavy-duty rubber gloves should always 
be used for cleaning dirty instruments'. I propose that mechanical washers should be used wherever practical, and that dirty instruments should be disinfected before cleaning - and please leave the choice of glove to the individual. The gloves should be discarded after each cleaning episode, so that the wearer's hands are not regularly bathed in a soup of contaminants which will seep through any un-noticed puncture of re-used gloves.

\section{S. W. White \\ Isle of White}

\section{RCTs}

Sir,- As a busy GDP I find myself inundated with various dental publications some of which go unread. Understandably it is important that we keep up to date with the latest advances and research. With so many articles one would think that RCTs (randomised controlled trials) would prove a God-send for the busy GDP.

Unfortunately this is not the case as having read many such articles I come away having learned little. Most of the conclusions or 'practice point' would read that the study 'suggests' something but further research is needed to develop evidence-based guidelines or recommendations. Surely the whole idea of these all-singing, all-dancing RCTs is to come to a solid conclusion or recommendation to enable the dentist to improve patient care and if RCTs do fall short are we then not doing an injustice to non-RCTs, case reports and anecdotal evidence if we continue to belittle what is often very useful work.

\section{J. J. Patel}

Slough

\section{A fair fee}

Sir,- With reference to Antony Townsend's reply to Dr Daniels' letter about the annual specialist list fee (BDJ 2003; 194: 466), I note in the most recent GDC Gazette (Winter 2002/3) that Mr Townsend states: 'The General Dental Council's principal duty is to patients..But the Council has other duties, of which one of the most important is fairness to registrants'. I fail to reconcile this statement with the facts. Presumably, GDC fairness excludes specialist orthodontists and oral surgeons, who have to continue to pay this exorbitant ASLF tax, in comparison to those listed on the other 11 'free' specialist lists.

\section{G. T. McIntyre}

Glasgow

\section{A dog's life}

Sir,- I began to read the letter 'Denture cleanliness' (BDJ 2003; 194: 354) with some foreboding and certainly after my tea as I was convinced that this case of dog devouring partial was going to be different.

Would it be that the dog swallowed it, the owner retrieved it on a walk a few days later and the denture still lives in its original home?

Much to my relief it was the traditional ending to the story which nevertheless was an interesting case.

\section{P. Williams}

Lowestoft

Sir,- There is currently a brand of dog biscuit, advertising that dogs eating their product have a $28 \%$ reduction in 'tartar'. The product even has a yellow ribbon printed diagonally across the corner with 'New! DentalCare' accompanied by a logo of a sparkling molar.

On studying the ingredients it seems that the active substance for calculus reduction is sodium hexametaphosphate. Should some enterprising manufacturer now include this in biscuits for human consumption?

\section{Yewe-Dyer}

Alton, Hants

\section{Tooth whitening}

Sir,- I would like to express my disappointment that as a dentist, I am not permitted to offer such a dramatic (relatively pain-free) non-invasive procedure, to the ever-increasing numbers of patients requesting it.

I would like to see the BDA push the DoH/EU/other bureaucrats on this matter, setting a deadline for the resolution of this 'classification' debacle.

We are after all, a patient-centred profession, are we not? I myself have used $16 \%$ carbamide peroxide in trays for ten nights, with excellent results. So too have a number of our other staff members. I trust that the profession will (very) soon be able to resume this service provision.

\section{F. Dean}

\section{Bournemouth}

Linda Wallace, Director, Professional Services Directorate, responds:- We agree with F. Dean's view on this. The BDA has been working with the UK Government and the EU Commission in an effort to resolve the issue and we hope this will be completed this year. 